\title{
Performance characterization of UV science cameras developed for the Chromospheric Lyman-Alpha Spectro-Polarimeter (CLASP)
}

\author{
UAHuntsville \\ 1,2Patrick Champey, ${ }^{2}$ Ken Kobayashi, ${ }^{2}$ Amy Winebarger, ${ }^{2}$ Jonathan Cirtin, ${ }^{2}$ David Hyde, \\ ${ }^{2}$ Bryan Robertson, ${ }^{2}$ Brent Beabout, ${ }^{2}$ Dyana Beabout, ${ }^{1}$ Mike Stewart
}

NASA

${ }^{1}$ University of Alabama in Huntsville, ${ }^{2}$ NASA Marshall Space Flight Center

\section{Abstract}

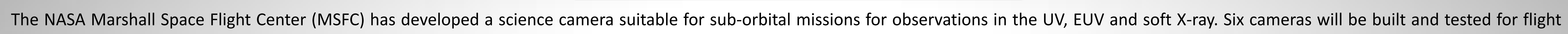

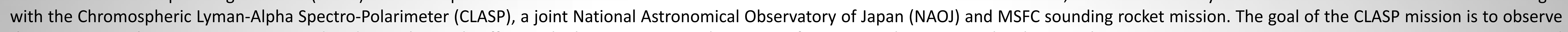

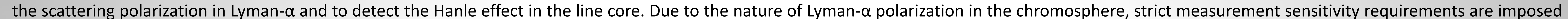

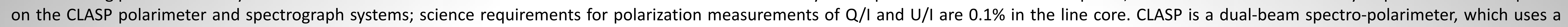

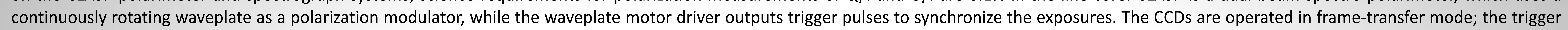

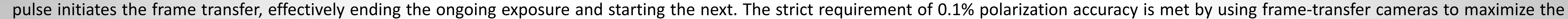

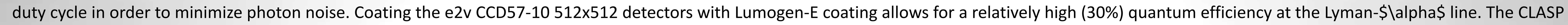

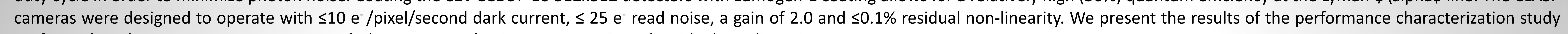
performed on the CLASP prototype camera; dark current, read noise, camera gain and residual non-linearity.

\section{Introduction}

The purpose of CLASP is to measure the linear polarization profiles caused by scattering processes and the Hanle effect in the Lya line. The magnetic field information can be obtained from the measured $Q / I$ and $U / I$ profiles themselves and mainly through detailed radiative transfer modeling of the observed Ly $\alpha$ intensity and polarization using the most advanced magnetohydrodynamic models of the solar atmosphere. This will provide, for the first time, a diagnostic tool for magnetic field measurements in the upper chromosphere and transition region.

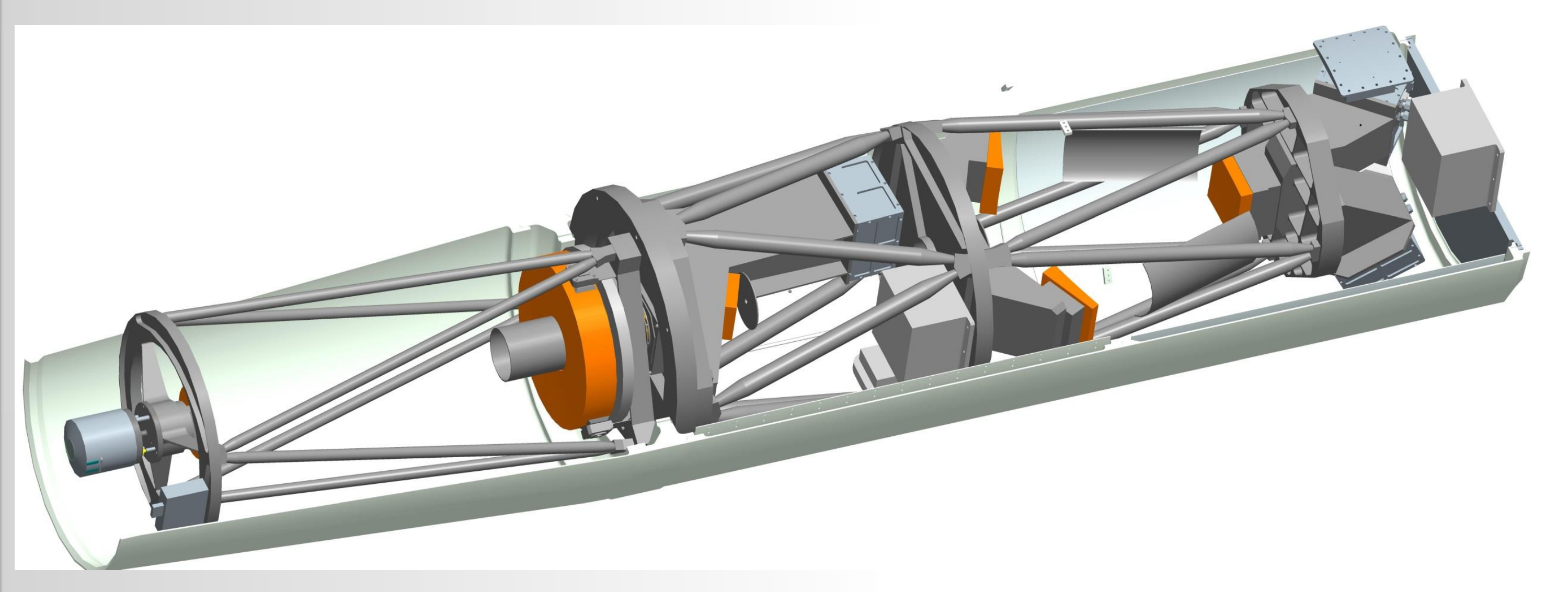

\section{CLASP Instrument}

- Cassegrain telescope, optimized for reflecting Lya line $(121.6 \mathrm{~nm})$

- Slit jaw imager for pointing verification

- The spectro-polarimeter produces two spectra simultaneously (corresponding to two orthogona polarization states) on two separate CCD cameras.

The rotation of the waveplate sends simultaneous trigger pulses to the spectrograph and polarization analyzer cameras to initiate frame transfer.

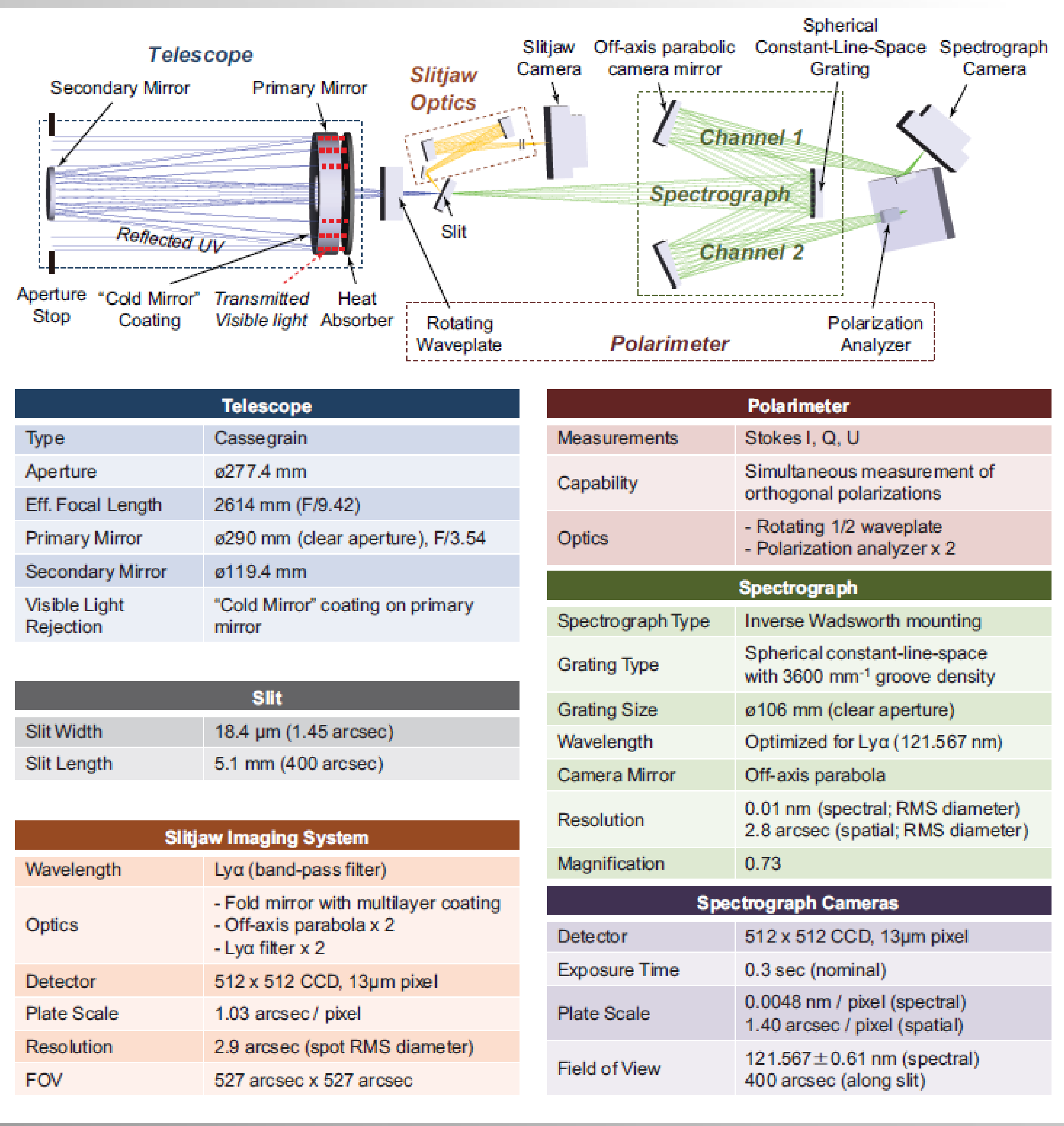

\section{CLASP Science Camera Characterization}

The CLASP mission requires the camera to operate with a stable gain $\left(2.0 \mathrm{e}^{-} / \mathrm{DN}\right)$, low dark current ( $\left.\leq 10 \mathrm{e}^{-} / \mathrm{pix} / \mathrm{sec}\right)$, low read noise $\left(\leq 25 \mathrm{e}^{-}\right)$and a residual non-linearity $\leq 0.1 \%$ to facilitate sensitive measurements of Ly $\alpha$ polarization modulation.

\section{Dark Current \& Read Noise}
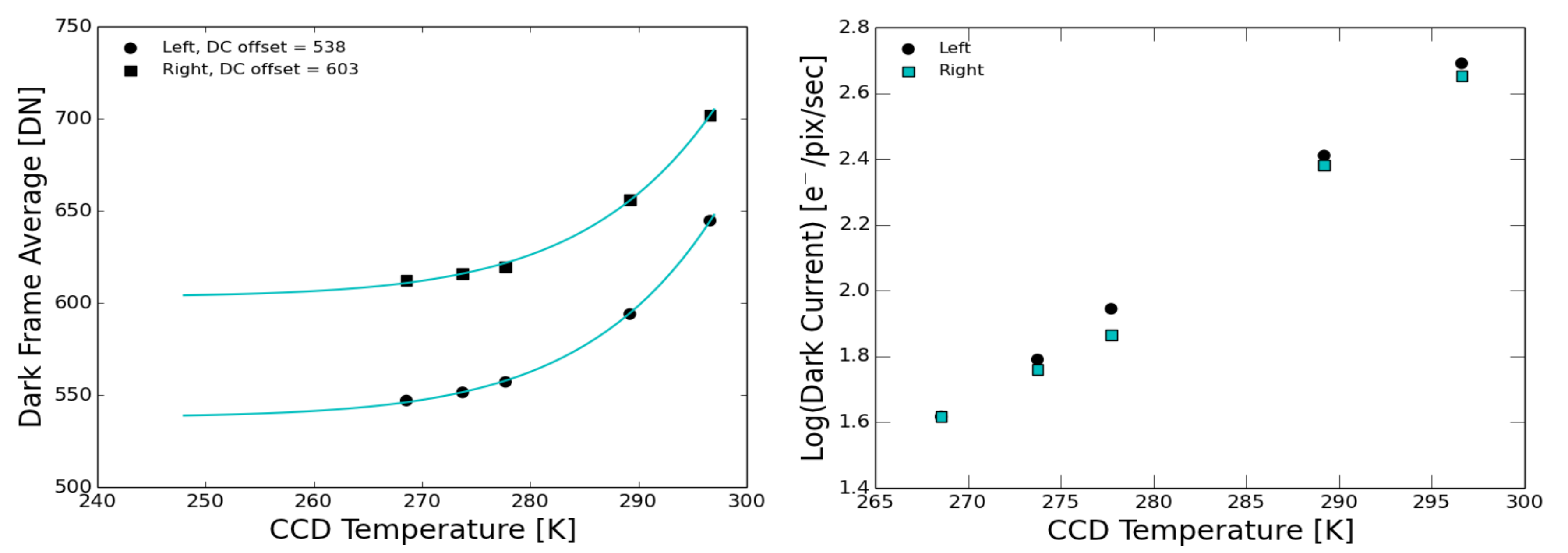

Calculated a dark current at $268 \mathrm{~K}$ of $41 \mathrm{e}-/ \mathrm{pix} / \mathrm{sec}$ for both left and right sides of the detector.

- Solving equation 1 for the flight set temperature of 253 , and applying equation 2 yields a dark current of $7.1 \mathrm{e}^{-} / \mathrm{pix} / \mathrm{sec}$ and $6.5 \mathrm{e}-/ \mathrm{pix} / \mathrm{sec}$ for left and right sides, respectively.

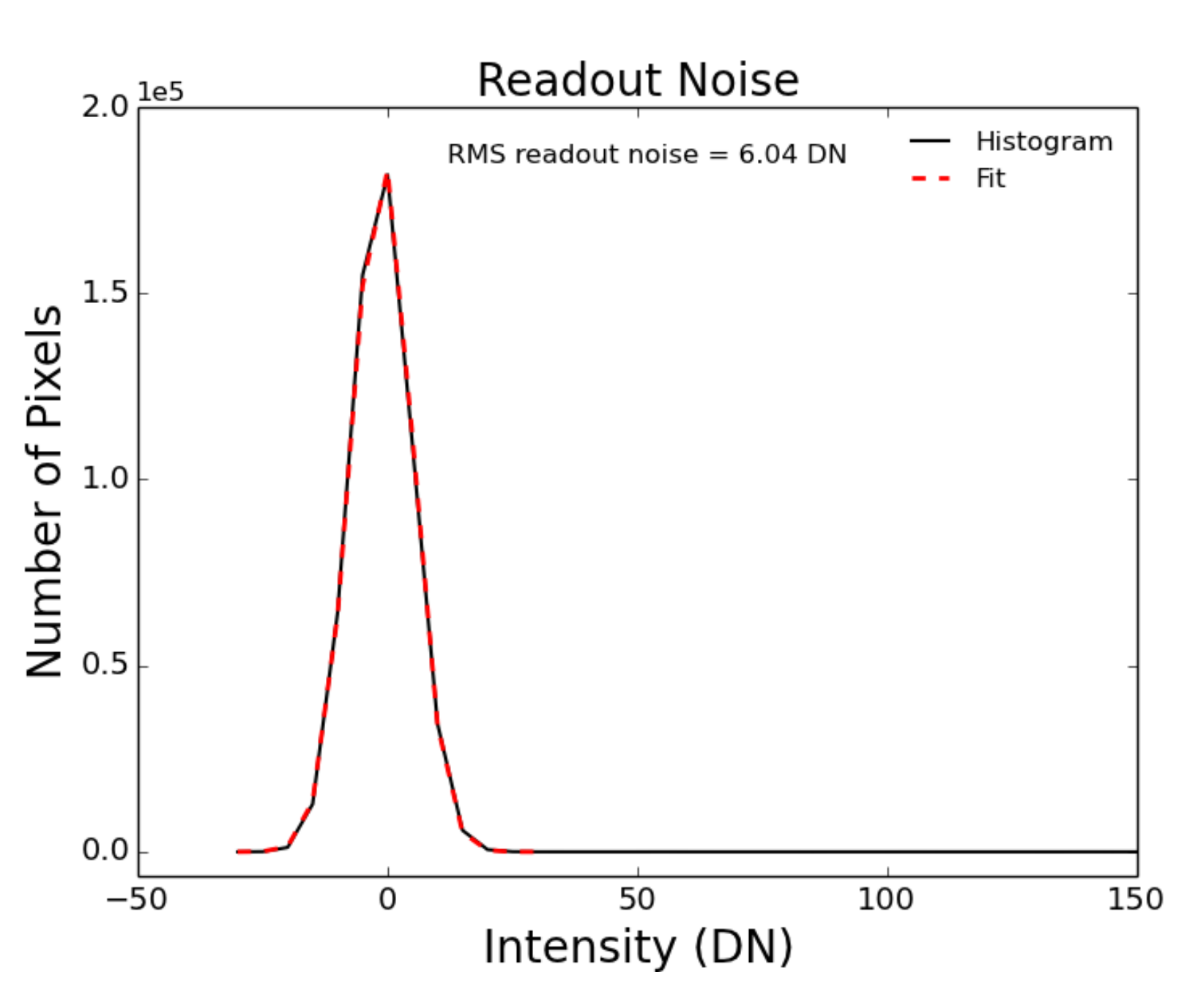

Read noise is measured by subtracting the master dark frame from a dark image, then calculating a histogram of the residual pixel values.

The histogram is fitted with a Gaussian function, and the width of that Gaussian is the read noise of the camera.

The CLASP requirement is a read noise $\leq 25 \mathrm{e}$

\section{Camera Gain}

- A $0.25 \mathrm{mCi}{ }^{55} \mathrm{Fe} \mathrm{X}$-ray source was used to measure the gain of the CCD and electronics chain.

${ }^{55} \mathrm{Fe} \mathrm{Mn} \mathrm{K}_{\alpha, \beta}$ lines produce a number of electrons proportional to their energies when absorbed by silicon.

The gain is determined by the location of the $\mathrm{Mn} \mathrm{K}_{\alpha, \beta}$ lines in the histogram of total ${ }^{55} \mathrm{Fe} \mathrm{X}$-rays detected.
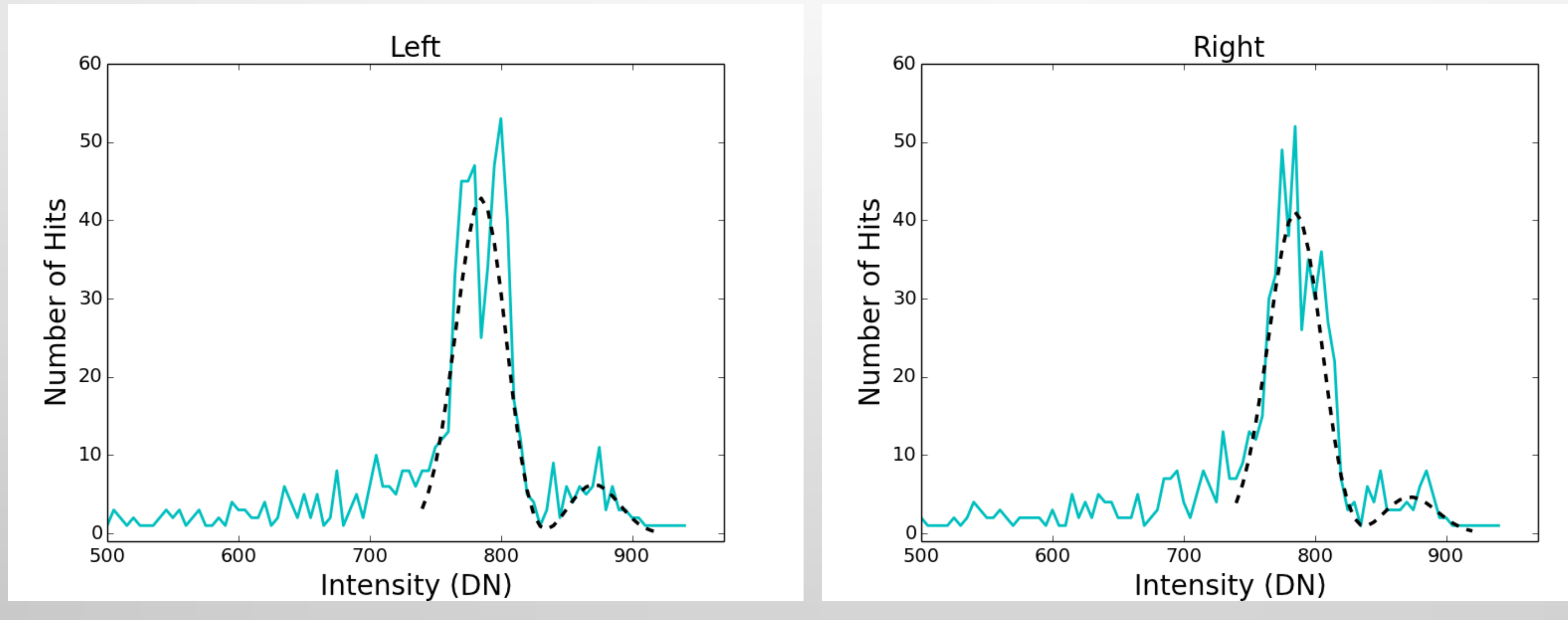

Linearity

- Standard flat fielding techniques were used to determine the linearity of the CLASP camera.

Variable output LED allowed the camera to expose from near dark levels, up to full saturation.

A photodiode was placed next to the CCD to measure relative incident photon flux by reading the output current via picoameter.

Residual non-linearity calculated by taking the ratio of the peak-to-valley deviation from the regression line, to the maximum intensity recorded in the dataset:
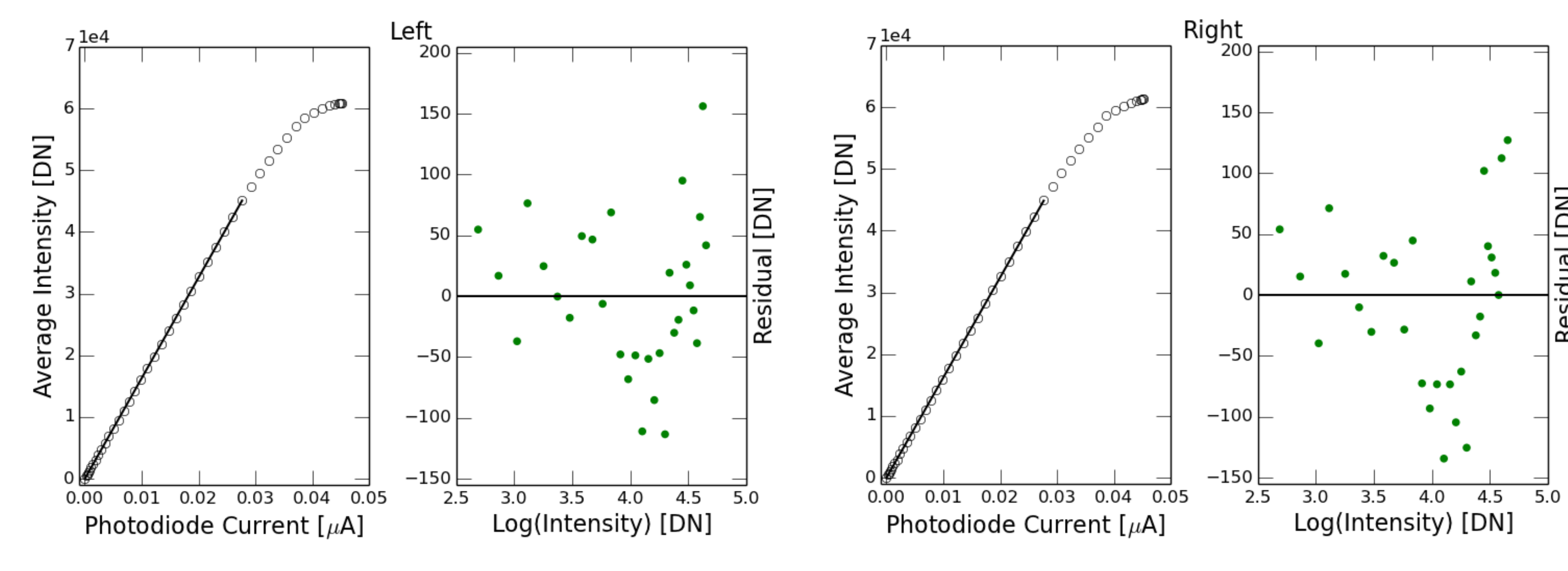

\section{Conclusion}

Testing the CLASP prototype camera in a thermally controlled environment proved to be a sufficient method for characterizing and verifying the prototype's performance.

- The dark current at $268 \mathrm{~K}\left(-5^{\circ} \mathrm{C}\right)$ was measured at $\mathrm{e}^{-} / \mathrm{pix} / \mathrm{sec}$ for both left and right sides of the CCD, while the dark current at the flight temperature of 253 $\mathrm{K}\left(-20^{\circ} \mathrm{C}\right)$ was calculated at $7.1 \mathrm{e}-/ \mathrm{pix} / \mathrm{sec}$ for the left side and $6.8 \mathrm{e}^{-} / \mathrm{pix} / \mathrm{sec}$ for the right side of the CCD.

- The average read noise was measured to be (XXX) (COMPARE TO REQUIREMENT).

The gain was determined to be 2.03 and 2.05 for the left and right sides, respectively.

Linearity of the prototype camera was determined to be $0.045 \%$ and $0.198 \%$ residual non-linearity for left and right sides, respectively.

Acknowledgements 